\title{
Weak coupling expansion of Yang-Mills theory on recursive infinite genus surfaces
}

\author{
Debashis Ghoshal, ${ }^{a}$ Camillo Imbimbo $^{b}$ and Dushyant Kumar ${ }^{a}$ \\ ${ }^{a}$ School of Physical Sciences, Jawaharlal Nehru University, \\ New Delhi 110067, India \\ ${ }^{b}$ Dipartimento di Fisica, Università degli studi di Genova and \\ INFN, Sezione di Genova, \\ Via Dodecaneso, 16146 Genova, Italy \\ E-mail: dghoshal@mail.jnu.ac.in, camillo.imbimbo@ge.infn.it, \\ sehrawat.dushyant@gmail.com
}

AbStRact: We analyze the partition function of two dimensional Yang-Mills theory on a family of surfaces of infinite genus. These surfaces have a recursive structure, which was used by one of us to compute the partition function that results in a generalized Migdal formula. In this paper we study the 'small area' (weak coupling) expansion of the partition function, by exploiting the fact that the generalized Migdal formula is analytic in the (complexification of the) Euler characteristic. The structure of the perturbative part of the weak coupling expansion suggests that the moduli space of flat connections (of the $\mathrm{SU}(2)$ and $\mathrm{SO}(3)$ theories) on these infinite genus surfaces are well defined, perhaps in an appropriate regularization.

Keywords: Field Theories in Lower Dimensions, Topological Field Theories, Nonperturbative Effects

ARXIV EPRINT: 1407.6380 


\section{Contents}

1 Introduction 1

2 Richards surfaces $\quad 4$

3 Yang-Mills theory on Riemann surfaces 5

$\begin{array}{lll}3.1 & \mathrm{SU}(2) \text { theory } & 6\end{array}$

$\begin{array}{lll}3.2 & \mathrm{SO}(3) \text { theory } & 7\end{array}$

4 Analysis of 2dYM on Richards surfaces $\quad 8$

4.1 Gauge group $\mathrm{SU}(2) \quad 9$

$\begin{array}{lll}\text { 4.1.1 Comparison at negative integer } \alpha & 10\end{array}$

$\begin{array}{ll}4.2 \text { Gauge group } \mathrm{SO}(3) & 11\end{array}$

$\begin{array}{lll}\text { 4.2.1 Comparison at negative integer } \alpha & 12\end{array}$

$\begin{array}{lll}5 & \mathrm{SU}(2) & 12\end{array}$

6 Conclusions 13

\section{Introduction}

Yang-Mills gauge field theory in two dimensions (2dYM) has been studied for a long time for its rich mathematical and physical content [1-4]. It also serves as a testing ground for new ideas. 2dYM and its quantum deformation have been found to be related to statistical systems, topological strings and even black holes [5-10]. Since gauge fields in two dimensions do not have any local propagating degrees of freedom, one would surmise that $2 \mathrm{dYM}$ is a topological quantum field theory, one that depends only on the global properties of two dimensional space-time. As a matter of fact it turns out that the theory is only 'almost' topological. Indeed, the lattice action on a triangulation of the surface leads to an exact expression [2] for the partition function (Euclidean path integral) that depends on the area of the surface, as well as its Euler characteristics. The area of the surface is the only 'non-topological' parameter, and it is really the gauge coupling constant.

The topological content of $2 \mathrm{dYM}$ was unravelled by Witten $[3,4]$ (see also the extensive review [11]), who reconsidered the model from the point of view of topological quantum field theories, which were developed at that time. The observation that the theory is indeed topological in the zero-coupling limit, led to the identification of the partition function of physical YM at non-zero coupling with the generating function of the correlators of certain 
BRST invariant observables of the topological YM. The topological supersymmetry underlying $2 \mathrm{dYM}$ allows the partition function to localize ${ }^{1}$ around the space of inequivalent flat gauge connections. The moduli space $\mathcal{M}_{g}$ of flat gauge connections, depends on the gauge group $G$ as well as the genus $g$ of the two dimensional surface (Euclidean space-time). The correspondence (between the topological and physical theory) means that the coefficients of the perturbative expansion of the partition function of physical $2 \mathrm{dYM}$ are related to the integrals of certain cohomology classes on $\mathcal{M}_{g}$. These integrals are very hard to compute directly for generic $g$ (and $G$ ), since the dimension $\mathcal{M}_{g}$ grows with the genus and its topology also becomes more intricate at higher genera. For this reason, the computation of these numbers using mathematical tools are limited. (Some relevant mathematical results on the moduli spaces are to be found in refs. [20-26].) The correspondence of ref. [4] allows for a computation using simpler 'physicists' method' for any $g$ and generic gauge groups. The moduli spaces $\mathcal{M}_{g}$ are also of considerable physical importance: as the phase space of Chern-Simons theory in three dimensions and in the description of WZW models, which form an important class of two dimensional conformal field theories. More generally these moduli spaces are low dimensional analogues of the moduli space of instantons of four dimensional Yang-Mills theory, the geometry of which is crucial for the understanding of the dynamics of (supersymmetric) gauge theories and their dualities in four dimensions.

Recently, one of us studied 2dYM on a class of surfaces of infinite genus. In particular, the recursive structure of these surfaces was exploited to arrive at a formula for the partition function [27]. We shall refer to these as Richards surfaces after Richards, who classified surfaces of infinite characteristics [28]. Strictly speaking, however, our surfaces are not among those in ref. [28], which are surfaces with 'ideal boundaries' at infinity, characterized by subsets of the Cantor set. On the other hand, the surfaces we consider, obtained from different compactifications, parametrized by two integers, of a surface in [28], have singularities in place of the 'ideal boundaries'. The partition function of $2 \mathrm{dYM}$ on these surfaces turns out to bear a surprising resemblance to the Migdal formula. Indeed, the Euler characteristic is replaced by a rational number, which may be thought of as the formal Euler characteristic of the recursive surface.

In the light of what we have reviewed above, one would expect that the perturbative expansion of the generalized Migdal formula of [27] encodes integrals of appropriate forms on the moduli space $\mathcal{M}\left(\overline{\mathcal{S}}_{g, p}\right)$ of flat connections on the Richards surfaces $\overline{\mathcal{S}}_{g, p}$ parametrized by the integers $g$ and $p$. The mathematical properties of this moduli space are, as yet, largely unknown. However we expect it to be infinite-dimensional. Therefore we can anticipate that the weak coupling expansion of the generalized Migdal formula of [27] contains exact information about (an infinite number of) integrals of appropriate forms on the infinite dimensional moduli spaces. The correspondence between the topological and physical 2dYM on Richard surfaces may, therefore, provide the first known example of a topological quantum theory which localizes on an infinite-dimensional moduli space

\footnotetext{
${ }^{1}$ Indeed 2 dYM was the first quantum field theory in the context of which the methods of non-abelian localization for quantum field theories were developed and tested. More recently the idea of localization has lead to enormous progress in our understanding of (supersymmetric) field theories in diverse dimensions. An incomplete set of references are [4, 12-19].
} 
in an exactly computable way. Moreover one expects $\mathcal{M}\left(\overline{\mathcal{S}}_{g, p}\right)$ to be relevant for higherdimensional gauge theories in the same way as the finite genus $\mathcal{M}_{g}$ is. An additional motivation to study $2 \mathrm{dYM}$ on Richard surfaces is that analytical results in this topic may give some insight to the old ideas $[29,30]$ concerning the significance of $2 \mathrm{~d}$ CFT on infinite genus surfaces for studying non-perturbative aspects of string theory.

In order to extract the intersection numbers on the moduli spaces of flat connections, one has to go from strong coupling, which is the regime of validity of the Migdal formula, to weak coupling, the regime where the correspondence with topological 2dYM holds. Even in the finite genus case this is not trivial to do. This was achieved by a trick in refs. [3, 4] by considering a certain derivative of Migdal's partition function with respect to the coupling constant. This results in a theta function which can be Poisson resummed to generate the weak coupling expansion. It turns out that the perturbative part of the weak coupling expansion stops at a finite order, which depends on $g$. This is a reflection of the finite dimensionality of $\mathcal{M}_{g}$. The weak coupling expansion also contains non-perturbative contributions which are not related to $\mathcal{M}_{g}$ but represent the contributions from the moduli spaces of non-flat instantons.

Since the trick of $[3,4]$ does not work for the Richards surfaces, we adopt a different strategy. We observe that the generalized Migdal formula derived in [27] can be analytically continued to complex values of the generalized Euler characteristics. In a suitable region of the complex plane (of the generalized Euler charateristics) the partition function is a sum over integer values of a function which admits a Fourier transform. This allows for a Poisson resummation of the generalized Migdal formula even for the Richards case. We perform this explicitly for the $\mathrm{SO}(3)$ and $\mathrm{SU}(2)$ theories - although the strategy may be applied to generic gauge groups - and derive explicit formulas for the invariants, which should be identified with the integrals on the infinite dimensional moduli spaces $\mathcal{M}\left(\overline{\mathcal{S}}_{g, p}\right)$, which are yet to be understood mathematically.

Our weak-coupling formula for YM on Richard surfaces also includes all the exponentially small, non-perturbative contributions, which are not related to $\mathcal{M}_{g}$, but represent the contributions to the partition function of moduli spaces of non-flat instantons. A detailed analysis of these terms is expected to shed light on the resurgence structure in a quantum field theory, however, we leave it as a project for the future.

In the following, we review the construction of a Richards surface and the generalized Migdal formula for the partition function of $2 \mathrm{dYM}$ on it [27]. We also briefly recapitulate the results from refs. [3, 4] on (finite genus) Riemann surfaces. We then perform the weak coupling expansion of the partition function on Richards surface. We find that, in this limit, the partition function can be separated into a part which is analytic and another which vanishes exponentially. (For the $\mathrm{SU}(2)$ case, there are also non-analytic terms attributable to singularities in the moduli space.) This parallels the case of surfaces of finite characteristics for which the analytic part is a polynomial. Indeed in such cases, the formal Euler characteristic coincides with the Euler characteristic, and our results coincide with those in refs. $[3,4]$, thanks to non-trivial identities of zeta functions. We will also compute the partition function (of the $\mathrm{SU}(2)$ theory for definiteness) with a fixed (nontrivial) holonomy along a boundary of the surface. This results in a generating function with two parameters. 

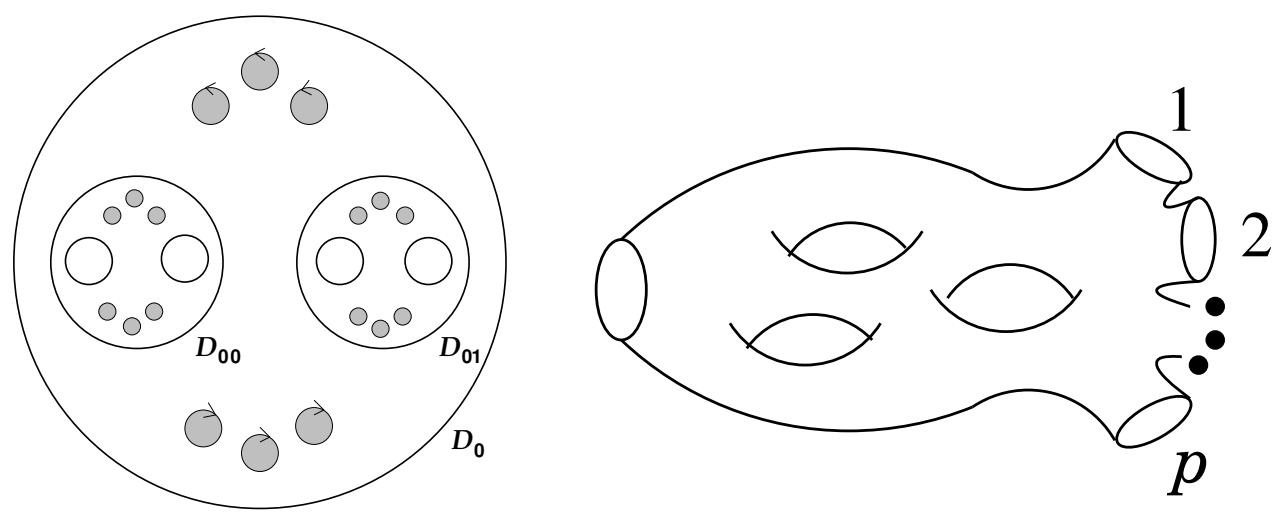

Figure 1. Left: iterative construction of a Richards surface starting with the disc $\mathcal{D}_{0}$. In this figure $p=2$ and $g=3$. Right: the basic building block of a Richards surface with $g=3$ and an arbitrary number of branchings.

\section{Richards surfaces}

In ref. [28], Richards gave a classification of noncompact surfaces, which have 'ideal boundaries' at asymptotic infinity labelled by a subset of the Cantor set. Recall that a Cantor set may be constructed by removing every alternate $\ell$ intervals out of the $(2 \ell+1)$ segments of $[0,1]$, for any positive integer $\ell$. This process is then repeated for every remaining intervals of the first set, and so on ad infinitum. The result is a totally disconnected space.

Motivated by the arguments in [28], one of us considered a family of surfaces, which we refer to as Richards surfaces. These may be thought of as compactifications of a surface in Richards' classification. The points in the set of ideal boundary, homeomorphic to the Cantor set, are singular points of these surfaces. Let us recall this construction. We start with a closed disc $\mathcal{D}_{0}$ containing the interval $[0,1]$, which in turn, contains the Cantor set. To be specific, we consider, $\ell=1$, for which we can take the discs $\mathcal{D}_{00}$ and $\mathcal{D}_{01}$ which contain the intervals $\left[0, \frac{1}{3}\right]$ and $\left[\frac{2}{3}, 1\right]$, respectively, and do not overlap. Let us attach $g$ handles to the complement of $\mathcal{D}_{00} \cup \mathcal{D}_{01}$ in $\mathcal{D}_{0}$. Equivalently, we remove $2 g$ disjoint discs in the complement and identify the boundaries of each pair preserving orientation. In the next step, perform similar operations in $\mathcal{D}_{00}$ and $\mathcal{D}_{01}$, and so on. The limit of this iterative process is an infinite genus surface which is made up of segments with $g$ handles and $p=2$ boundaries, to each of which is attached another similar segment. We refer to it as a Richards surface with parameters $p$ and $g$ (see figure 1).

The limiting sets $\mathcal{D}_{000 \ldots}, \mathcal{D}_{001 \ldots}, \mathcal{D}_{010 \ldots}$, etc. contain the singular points of this surface. In ref. [28], where the points of the Cantor set are removed from $\mathcal{D}_{0}$ from the beginning, these limiting sets would have been the 'ideal boundaries' instead. The noncompact surface $\mathcal{S}$ obtained by the latter procedure can be shown to be independent of the choices of integers $g$ and $p$, and therefore, results in a unique (upto homeomorphism) surface. Therefore, our surfaces $\overline{\mathcal{S}}_{g, p}$ are to be thought of as compactifications of it.

In ref. [27] the recursive nature of these surfaces was used to compute the partition function of $2 \mathrm{dYM}$ for gauge group $G$ : for a Richards surface $\bar{\Sigma}_{g, p}$ given by the parameters 
$g$ (for the numbers of handles attached to each building block) and $p$ (for the number of branchings at each step) is

$$
Z_{\infty}^{(g, p)}\left(\epsilon ; U_{0}\right)=\sum_{R}(\operatorname{dim} R)^{1+\frac{2 g}{p-1}} e^{-4 \pi^{2} \epsilon C_{2}(R)} \chi_{R}\left(U_{0}\right)
$$

where the sum is over all irreducible representations $R$ of the Lie group $G, C_{2}(R)$ and $\chi(R)$ are, respectively, the second Casimir operator and the character corresponding to $R$ and the dimensionless parameter $\epsilon=a \tilde{g}^{2} / 4 \pi^{2}$ is given in terms of the (regularized) area $a$ of the surface and the coupling constant $\tilde{g}^{2}$ of the gauge theory. We have also put a holonomy $U$ along the boundary of the surface. (This is the boundary of the initial disc $\mathcal{D}_{0}$.) This formula is not applicable to $p=1$, which results in a surface known as the Loch Ness monster. However, it is consistent with the known results for the special cases (i) $g=0$ (a disc) and (ii) $p=0$ (a surface of finite genus $g$ ).

The partition function of Richards surface is remarkably similar to the Migdal formula for surfaces with finite genus [2]. The main difference is that the power of $\operatorname{dim} R$ is fractional. In fact, being the formal sum of the characteristics of its buildings blocks, it may be thought of as the Euler characteristic of the Richards surface.

We see from the partition function (2.1) above, that Richards surfaces with different parameters $g$ and $p$ have generically different partition functions. Hence we may conclude (at the level of rigour used by physicists) that they are topologically inequivalent.

\section{Yang-Mills theory on Riemann surfaces}

Let us recall the analysis of the partition function of $2 \mathrm{dYM}$ in refs. [3, 4] to extract intersection numbers of cycles in the moduli space of flat connections on a Riemann surface. We start with the partition function of a surface of genus $g$ and the number of boundary components $p$ :

$$
Z_{(g, p)}\left(\epsilon ; U_{1}, \cdots, U_{p}\right)=\sum_{R}(\operatorname{dim} R)^{2-2 g-p} e^{-4 \pi^{2} \epsilon C_{2}(R)} \chi_{R}\left(U_{1}\right) \cdots \chi_{R}\left(U_{p}\right)
$$

where $\epsilon=\frac{a \tilde{g}^{2}}{4 \pi^{2}}$ is a dimensionless parameter defined earlier. The holonomies $U_{1}, \cdots, U_{p}$ are associated with the $p$ boundaries, and the second Casimir invariant $C_{2}(R)$ corresponds to the representation $R$. This partition function comes from integrating the exponential of the Yang-Mills action functional over the space of gauge inequivalent connections with specified holonomies along the given boundaries.

In the limit of $\epsilon \rightarrow 0$, the most dominant contribution to the partition function is from the integration over the space of flat connections. Thus, in this limit, one can expect the partition function to contain some topological information about the moduli space. This indeed is the case. The small area expansion of the partition function around $\epsilon=0$ consists of a polynomial part (also in addition, possibly some terms with fractional powers of $\epsilon$ ), and a part that is exponentially suppressed. The coefficients of the polynomial are interpreted as the intersection numbers of the homology cycles on the moduli space of flat connections. Let us recall the case of $\mathrm{SU}(2)$ and $\mathrm{SO}(3)$ groups, for which these computations have been performed independently [22]. 


\section{$3.1 \mathrm{SU}(2)$ theory}

Only a trivial principal SU(2) bundle is possible for a closed surface $\Sigma$ of genus $g$. Let us denote it as $E$. The exponential of the Yang-Mills action integrated (with respect to the symplectic measure) over the space of connections on $E$ gives the following partition function

$$
Z_{g}(\epsilon)=\frac{1}{\left(2 \pi^{2}\right)^{g-1}} \sum_{n=1}^{\infty} n^{2-2 g} \exp \left(-\epsilon \pi^{2} n^{2}\right),
$$

This is the Migdal formula for $\mathrm{SU}(2)$, normalized by the volume of the gauge group as in refs. $[3,4]$.

Let $\mathcal{M}$ denote the moduli space of (gauge equivalence classes of) flat $\mathrm{SU}(2)$ connections on $E$. The partition function (3.2) for $g \geq 1$ is related to the integration of the closed differential forms $\omega$ and $\Theta$, of degree 2 and 4 respectively, on $\mathcal{M}$. Of these, $\omega$ is the pull-back on $\mathcal{M}$ of the natural symplectic form

$$
\omega(\delta A, \delta A)=\int_{\Sigma} \operatorname{Tr} \delta A \wedge \delta A
$$

which exists on the space of $\mathrm{SU}(2)$ connections on $\Sigma$. On the other hand, as explained in [4], $\Theta$ has a natural interpretation in the two dimensional topological Yang-Mills theory. The topological YM gauge multiplet contains, in addition to the connection field $A_{\mu}(x) d x^{\mu}$ and its fermionic super-partner $\psi_{\mu}(x) d x^{\mu}$ of ghost number 1, a scalar field $\phi(x)$ of ghost number 2, all in the adjoint representation of the gauge group. The cohomology class of $\Theta$ is associated to the BRST invariant local observable $\operatorname{Tr} \phi^{2}(x)$. It turns out that for fixed non-negative integers $k \leq \frac{1}{4} \operatorname{dim} \mathcal{M}$ and $s=\frac{1}{2} \operatorname{dim} \mathcal{M}-k$,

$$
\frac{1}{(s-k) !} \int_{\mathcal{M}} \omega^{s-k} \wedge \Theta^{k}=\left.2 \frac{d^{k}}{d \epsilon^{k}} Z_{g}(\epsilon)\right|_{\epsilon=0},
$$

whenever the l.h.s. and r.h.s. of this equation make sense. The factor of 2 on the r.h.s. is the number of elements in the centre of $\mathrm{SU}(2)$. The above equation can be written in a compact form as

$$
\int_{\mathcal{M}} \exp (\omega+\epsilon \Theta)=2 Z_{g}(\epsilon) \quad \text { (modulo exponentially small terms). }
$$

The expansion in Migdal formula eq. (3.2) is useful for $\epsilon>0$ large. To extract the $\epsilon \rightarrow 0^{+}$behaviour of the partition function and make contact with the topological numbers in eq. (3.4) one cannot simply expand the exponentials in the series and take the limit, since this would result in a divergent expression. The trick used in refs. [3, 4] is to consider the derivative

$$
\begin{aligned}
\frac{d^{g-1}}{d \epsilon^{g-1}} Z_{g}(\epsilon) & =\frac{(-1)^{g}}{2^{g-1}} \sum_{n=1}^{\infty} \exp \left(-\epsilon \pi^{2} n^{2}\right) \\
& =\frac{(-1)^{g-1}}{2^{g}}\left[\sum_{n=-\infty}^{\infty} \exp \left(-\epsilon \pi^{2} n^{2}\right)-1\right],
\end{aligned}
$$


and to make use of the Poisson resummation formula

$$
\sum_{n=-\infty}^{\infty} f(n)=\sum_{k=-\infty}^{\infty} \tilde{f}(k)
$$

(where $\tilde{f}$ is the Fourier transform of $f$ ) to obtain

$$
\begin{aligned}
\frac{d^{g-1}}{d \epsilon^{g-1}} Z_{g}(\epsilon) & =\frac{(-1)^{g-1}}{2^{g}}\left[\frac{1}{\sqrt{\pi \epsilon}} \sum_{k=-\infty}^{\infty} \exp \left(-\frac{\pi^{2}}{\epsilon} k^{2}\right)-1\right] \\
& =\frac{(-1)^{g-1}}{2^{g}}\left(\frac{1}{\sqrt{\pi \epsilon}}-1\right)+\text { exponentially small terms. }
\end{aligned}
$$

This determines $Z_{g}(\epsilon)$ up to a polynomial $P^{(g-2)}(\epsilon)$ in $\epsilon$ of degree $g-2$ (for $g \geq 2$ ).

It is the polynomial which contains the information about the intersection numbers (3.4). In order to determine the coefficients of this polynomial, one can evaluate the first $k$ derivatives of the Migdal formula at $\epsilon=0$

$$
\left.\frac{d^{k}}{d \epsilon^{k}} Z_{g}(\epsilon)\right|_{\epsilon=0}=\frac{(-1)^{k}}{2^{g-1} \pi^{2(g-1-k)}} \sum_{n=1}^{\infty} \frac{1}{n^{2(g-1-k)}} \quad \text { for } \quad k<g-1 .
$$

in which the series on the r.h.s. are convergent for $k<g-1$. In this way, one finds that

$$
Z_{g}(\epsilon)=\sum_{k=0}^{g-2} a_{k} \epsilon^{k}+a_{g-\frac{3}{2}} \epsilon^{g-\frac{3}{2}}+a_{g-1} \epsilon^{g-1}+\text { exponentially small terms, }
$$

where the coefficients are

$$
\begin{aligned}
a_{k} & =\frac{(-1)^{k}}{k ! 2^{g-1} \pi^{2 g-2-2 k}} \zeta(2 g-2-2 k) \quad \text { for } k=0,1, \cdots, g-2, \\
a_{g-\frac{3}{2}} & =\frac{(-1)^{g-1}}{2 \sqrt{\pi}(2 g-3) ! !} \text { and } a_{g-1}=\frac{(-1)^{g}}{2^{g}(g-1) !} .
\end{aligned}
$$

Note that the coefficient of the nonanalytic term, $a_{g-\frac{3}{2}}$, is nonzero, which is a manifestation of the fact that the moduli space of $\mathrm{SU}(2)$ flat connections is singular. This is due to that fact that $\mathrm{SU}(2)$ has a nontrivial centre and hence the action of $\mathrm{SU}(2)$ gauge group on the space of flat connections is not free. One can read off the intersection numbers on $\mathcal{M}$, given in eq. (3.4), from eqs. (3.10) and (3.11). (We note parenthetically that a factor of -1 is missing from eqs. (4.52) and (4.53) in [4] due to a typographical error. The corresponding expressions are eqs. (3.6) and (3.8) above.)

\section{$3.2 \mathrm{SO}(3)$ theory}

The calculations for $\mathrm{SO}(3)$ are similar to that for $\mathrm{SU}(2)$ except for a few important differences. First, for a closed surface $\Sigma$ of genus $g \geq 1$, two topologically inequivalent $\mathrm{SO}(3)$ principal bundles $E^{\prime}(1)$ and $E^{\prime}(-1)$ are possible. Of these, $E^{\prime}(1)$ is trivial and lifts to an $\mathrm{SU}(2)$ bundle on $\Sigma$. On the other hand $E^{\prime}(-1)$ is nontrivial and can be lifted to an $\mathrm{SU}(2)$ bundle $E_{p}$ only on the complement $\Sigma-\{p\}$ of a point $p \in \Sigma$. If we choose a flat 
$\mathrm{SU}(2)$ connection $A$ on $E_{p}$ then the holonomy of this connection about $p$ would be -1 , independent of the choice of $A$ and $p$.

Let $u= \pm 1$, then the partition function of $\mathrm{SO}(3)$ Yang-Mills theory on the space of connections on $E^{\prime}(u)$ is given by

$$
\widetilde{Z}_{g}(\epsilon ; u)=\frac{1}{2\left(8 \pi^{2}\right)^{g-1}} \sum_{n=1}^{\infty} n^{2-2 g} \lambda_{n}\left(u^{-1}\right) \exp \left(-\epsilon \pi^{2} n^{2}\right),
$$

where $\lambda_{n}\left(u^{-1}\right)=\chi_{n}\left(u^{-1}\right) / n$ in which $\chi_{n}\left(u^{-1}\right)$ is the trace of $u^{-1}$ in the representation of dimension $n$. The sum is over all the representations of $\mathrm{SU}(2)$ instead of only those of $\mathrm{SO}(3)$. However, the sum $\widetilde{Z}_{g}(\epsilon)=\widetilde{Z}_{g}(\epsilon ; 1)+\widetilde{Z}_{g}(\epsilon ;-1$ ) (which, in principle, is the full $\mathrm{SO}(3)$ partition function) will have contribution only from the irreducible representations of $\mathrm{SO}(3)$.

Secondly, let $\mathcal{M}^{\prime}(-1)$ denote the moduli space of (gauge equivalent classes of) flat $\mathrm{SO}(3)$ connections $^{2}$ for the bundle $E^{\prime}(-1)$. The partition function eq. (3.12) for $g \geq 1$ is related to the integration of the differential forms $\omega$ and $\Theta$ on $\mathcal{M}^{\prime}(-1)$ in the following way [4]. For fixed non-negative integers $k \leq \frac{1}{4} \operatorname{dim} \mathcal{M}^{\prime}(-1)$ and $s=\frac{1}{2} \operatorname{dim} \mathcal{M}^{\prime}(-1)-k$,

$$
\frac{1}{(s-k) !} \int_{\mathcal{M}^{\prime}(-1)} \omega^{s-k} \wedge \Theta^{k}=\left.\frac{d^{k}}{d \epsilon^{k}} \widetilde{Z}_{g}(\epsilon ;-1)\right|_{\epsilon=0},
$$

where there is not any factor of 2 this time because the centre of $\mathrm{SO}(3)$ is trivial. The numbers $\left.\frac{d^{k}}{d \epsilon^{k}} \widetilde{Z}(\Sigma, \epsilon ;-1)\right|_{\epsilon=0}$ can be calculated in exactly the same way as for $\mathrm{SU}(2)$ : for $k \leq$ $(g-2)$ directly from eq. (3.12), and for $k \geq(g-1)$ by using Poisson resummation. We have

$$
Z_{g}(\epsilon)=\sum_{k=0}^{g-1} a_{k} \epsilon^{k}+\text { exponentially small terms, }
$$

where the coefficients are

$$
a_{k}=(-1)^{k} \frac{\left(1-2^{2 g-3-2 k}\right) \zeta(2 g-2-2 k)}{k ! 2^{3 g-3}(2 \pi)^{2 g-2-2 k}} .
$$

Notice that the r.h.s. does not have any term with fractional powers of $\epsilon$ as $\mathcal{M}^{\prime}(-1)$ is nonsingular, and $a_{0}$ is its symplectic volume.

\section{Analysis of 2dYM on Richards surfaces}

Richards surfaces have a self-similar structure which allows one to compute the partition function of Yang-Mills theory on them. The partition function for a surface $\overline{\mathcal{S}}_{g, p}$ is given in eq. (2.1). As we have pointed out earlier, the only difference from the partition function of finite genus surfaces, eq. (3.1), is that the power of $\operatorname{dim} R$ is in general a positive fraction (which may be thought of as the formal Euler characteristic). In the following we will work with the assumption that the partition function of $2 \mathrm{dYM}$ on Richards surfaces would allow for an interpretation as a generating function of intersection numbers on moduli space of flat connections of principal $G$-bundle on Richards surface, exactly analogous to the finite genus surfaces. As in the previous section, we will consider the cases of $\mathrm{SU}(2)$ and $\mathrm{SO}(3)$.

\footnotetext{
${ }^{2}$ The case of $\mathcal{M}^{\prime}(1)$ is exactly same as that for $\mathrm{SU}(2)$.
} 


\subsection{Gauge group $\mathrm{SU}(2)$}

The extension of the SU(2) Migdal formula to the infinite genus Richards surfaces [27] takes the form ${ }^{3}$

$$
Z(\alpha ; \epsilon)=\sum_{n=1}^{\infty} n^{\alpha} \exp \left(-\epsilon \pi^{2} n^{2}\right)
$$

where

$$
\alpha=2+\frac{2 g}{p-1}
$$

is now a rational number. It is reasonable to suppose that formula (4.1) encodes some kind of topological information by means of an equation analogous to eq. (3.5), involving integration of classes on the infinite dimensional moduli space $\mathcal{M}\left(\overline{\mathcal{S}}_{g, p}\right)$ of flat connections on the Richards surfaces with fixed $g$ and $p$. It would be interesting therefore to determine the behaviour of the function in eq. (4.1) near $\epsilon=0$. To obtain this, obviously we cannot exploit the same trick of refs. [3, 4], as we reviewed in the previous section: this trick involved taking the $g-1$ derivatives of the partition function with respect to $\epsilon$, which works only when $\alpha=(2-2 g)$ is a negative integer. We will therefore adopt a different strategy. Let us first of all extend $\alpha$ to the complex plane, and then extend the sum (4.1) over all integers as

$$
Z(\alpha ; \epsilon)=\frac{1}{\left(1+e^{i \pi \alpha}\right)} \sum_{n=-\infty}^{\infty} n^{\alpha} \exp \left(-\epsilon \pi^{2} n^{2}\right) .
$$

At this point, for $\alpha$ in a suitable region of the complex plane, we can use the Poisson resummation formula, eq. (3.7), with

$$
f(x)=x^{\alpha} e^{-\epsilon \pi^{2} x^{2}}
$$

Using the fact that, for $\Re(\alpha)>-1$,

$$
\begin{aligned}
\int_{-\infty}^{\infty} d x x^{\alpha} e^{-\epsilon \pi^{2} x^{2}-2 \pi i k x} & \\
=\frac{\epsilon^{-1-\frac{\alpha}{2}}}{2 \pi^{\alpha+1}}[ & {\left[\left(1+(-1)^{\alpha}\right) \sqrt{\epsilon} \Gamma\left(\frac{\alpha+1}{2}\right){ }_{1} F_{1}\left(\frac{\alpha+1}{2}, \frac{1}{2} ;-\frac{k^{2}}{\epsilon}\right)\right.} \\
& \left.\quad-2 i\left(1-(-1)^{\alpha}\right) k \Gamma\left(\frac{\alpha}{2}+1\right){ }_{1} F_{1}\left(\frac{\alpha}{2}+1, \frac{3}{2} ;-\frac{k^{2}}{\epsilon}\right)\right]
\end{aligned}
$$

where ${ }_{1} F_{1}(a, b ; z)$ is the Kummer confluent hypergeometric function defined by the series $\sum_{m=1}^{\infty} \frac{(a)_{m} z^{m}}{(b)_{m} m !}$, in which $(a)_{m}$ and $(b)_{m}$ are Pochhammer symbols, we obtain

$$
Z(\alpha ; \epsilon)=\frac{1}{2 \pi^{1+\alpha}} \Gamma\left(\frac{1+\alpha}{2}\right) \epsilon^{-(1+\alpha) / 2} \sum_{k=-\infty}^{\infty}{ }_{1} F_{1}\left(\frac{1+\alpha}{2}, \frac{1}{2} ;-\frac{k^{2}}{\epsilon}\right)
$$

\footnotetext{
${ }^{3}$ We have ignored the equivalent of the normalization factor $\left(2 \pi^{2}\right)^{g-1}$ in eq. (3.2), as the exact geometric analogue of it is not yet understood. The same applies to the eqs. (3.12) and (4.12) for $\mathrm{SO}(3)$, which has an additional factor of 2 .
} 
Since we want to know the behaviour of $Z(\epsilon, \alpha)$ near $\epsilon=0$, we use the asymptotic expansion

$$
{ }_{1} F_{1}(a, b ; z) \stackrel{z \rightarrow-\infty}{\longrightarrow} \frac{\Gamma(b)}{\Gamma(b-a)} \sum_{m=0}^{\infty} \frac{(a)_{m}(a-b+1)_{m}}{m !}(-z)^{-m-a}+\mathcal{O}\left(e^{z}\right),
$$

of the function ${ }_{1} F_{1}(z)$ for all $k \neq 0$ terms of the series above to write

$$
\begin{aligned}
Z(\alpha ; \epsilon)= & \frac{1}{2 \pi^{1+\alpha}} \Gamma\left(\frac{1+\alpha}{2}\right) \epsilon^{-\frac{1+\alpha}{2}} \\
& +\frac{1}{2 \pi^{1+\alpha}} \Gamma\left(\frac{1+\alpha}{2}\right) \frac{\sqrt{\pi}}{\epsilon^{\frac{1+\alpha}{2}}} \sum_{k \neq 0} \frac{1}{\Gamma\left(-\frac{\alpha}{2}\right)}\left(\frac{k^{2}}{\epsilon}\right)^{-\frac{1+\alpha}{2}} \sum_{m=0}^{\infty} \frac{\left(\frac{1+\alpha}{2}\right)_{m}\left(\frac{2+\alpha}{2}\right)_{m}}{m !}\left(\frac{k^{2}}{\epsilon}\right)^{-m} \\
& + \text { exponentially small terms. }
\end{aligned}
$$

We can now sum over $k$ to get the final expression for $Z(\epsilon)$ (modulo exponentially small terms):

$$
\begin{aligned}
Z(\alpha ; \epsilon)= & \frac{\Gamma\left(\frac{1+\alpha}{2}\right)}{2 \pi^{1+\alpha} \epsilon^{\frac{1+\alpha}{2}}}+\frac{\Gamma\left(\frac{1+\alpha}{2}\right) \sqrt{\pi}}{\pi^{1+\alpha} \Gamma\left(-\frac{\alpha}{2}\right)} \sum_{m=0}^{\infty} \frac{\left(\frac{1+\alpha}{2}\right)_{m}\left(\frac{2+\alpha}{2}\right)_{m}}{m !} \zeta(2 m+1+\alpha) \epsilon^{m} \\
& + \text { exponentially small terms. }
\end{aligned}
$$

The first term in this expression has a fractional negative power of $\epsilon$. We expect this to be related to the singularities of the purported moduli space owing to the nontrivial centre of the $\mathrm{SU}(2)$, exactly as in the case of Riemann surfaces of finite genus for which $\alpha$ is an even (negative) integer. We will confirm this interpretation in the next subsections, where we will show that the non-analytic terms of this kind are absent for both the $\mathrm{SO}(3)$ case (in the sector with non-trivial monopole charge) and the case when a non-trivial holonomy is inserted at a boundary/puncture on the surface.

The infinite sum in (4.9) is the part of the partition function that is analytic in $\epsilon$. The coefficients

$$
a_{m}=\frac{\Gamma\left(\frac{1+\alpha}{2}\right)}{\pi^{\frac{1}{2}+\alpha} \Gamma\left(-\frac{\alpha}{2}\right)} \frac{\left(\frac{1+\alpha}{2}\right)_{m}\left(\frac{2+\alpha}{2}\right)_{m}}{m !} \zeta(2 m+1+\alpha), \quad m=0,1, \cdots
$$

provide a generalization of the intersection numbers in eq. (3.11), to the Richards Surface.

The part of $Z(\alpha ; \epsilon)$ that is not analytic in $\epsilon$, consists of exponentially small terms. These are calculated explicitly in eq. (4.6) and can be studied systematically from the asymptotic expansion of the confluent hypergeometric function ${ }_{1} F_{1}$. They have the structure of instanton contributions of the form $e^{-\pi^{2} k^{2} / \epsilon}$, just as in the finite genus case.

\subsubsection{Comparison at negative integer $\alpha$}

For the analytic continuation in $\alpha$ to be consistent, one would expect to recover the results of ref. [4] by setting $\alpha=2-2 g$ in the expression above. This is indeed the case. The Pochhammer symbol $\left(\frac{2+\alpha}{2}\right)_{m}$ vanishes for all $m \geq g-1$, therefore the power series in $\epsilon$ terminates. However, for $m=(g-1)$, the zeta function has a simple pole, which compensates for the zero from the Pochhammer symbol and gives a finite contribution, which agrees with the coefficient $a_{g-1}$ in eq. (3.10). Also the coefficient of $\epsilon^{-\frac{1+\alpha}{2}}$ in eq. (3.10) 
agrees with the one in (3.11) when $\alpha=2-2 g$. We are left with a polynomial of degree $(g-2)$ in $\epsilon$ which is identical to the corresponding polynomial in eq. (3.10). The matching, however, is not trivial, as it involves the Riemann functional equation

$$
\zeta(s)=2^{s} \pi^{s-1} \sin \left(\frac{\pi s}{2}\right) \Gamma(1-s) \zeta(1-s)
$$

for the Riemann zeta function, as well as identities for the $\Gamma$-function, the use of which shows that

$$
\begin{aligned}
& \frac{\sqrt{\pi} \Gamma\left(\frac{3}{2}-g\right)}{\pi^{3-2 g} \Gamma(g-1)} \sum_{m=0}^{g-2} \frac{\left(\frac{3}{2}-g\right)_{m}(2-g)_{m}}{m !} \zeta(2 m+3-2 g) \epsilon^{m} \\
& \quad=\frac{\sqrt{\pi}(-1)^{g-1}}{\pi^{2-2 g} \Gamma(g-1) \Gamma\left(g-\frac{1}{2}\right)} \sum_{m=0}^{g-2} \frac{\Gamma(2 g-2) \zeta(2 m+3-2 g)}{m ! 2^{2 m} \Gamma(2 g-2 m-2)} \epsilon^{m} \\
& =\sum_{m=0}^{g-2} \frac{(-1)^{m} \pi^{2 m} \zeta(2 g-2 m-2)}{m !} \epsilon^{m},
\end{aligned}
$$

in agreement with eq. (3.11). (The coefficients match precisely when the volume factor mentioned in footnote 3 is taken into account.)

\subsection{Gauge group $\mathrm{SO}(3)$}

In this case we start with the partition function in the sector with monopole charge $u=-1$

$$
\widetilde{Z}(\alpha ; \epsilon ;-1)=\sum_{n=1}^{\infty}(-1)^{n} n^{\alpha} \exp \left(-\epsilon \pi^{2} n^{2}\right),
$$

which we rewrite as

$$
\widetilde{Z}(\alpha ; \epsilon ;-1)=\frac{1}{\left(1+e^{i \pi \alpha}\right)} \sum_{n=-\infty}^{\infty} n^{\alpha} \exp \left(-\pi^{2} \epsilon n^{2}+i \pi n\right) .
$$

After a Poisson resummation, it takes the form

$$
\widetilde{Z}(\alpha ; \epsilon ;-1)=\frac{1}{2\left(\pi^{2} \epsilon\right)^{\frac{1+\alpha}{2}}} \Gamma\left(\frac{1+\alpha}{2}\right) \sum_{k=-\infty}^{\infty}{ }_{1} F_{1}\left(\frac{1+\alpha}{2}, \frac{1}{2},-\frac{\left(k-\frac{1}{2}\right)^{2}}{\epsilon}\right) .
$$

Now, for small $\epsilon$, we use the asymptotic expansion eq. (4.7) of the confluent hypergeometric function to write the $k$-th term in the series as

$$
\frac{\sqrt{\pi}}{\Gamma\left(-\frac{\alpha}{2}\right)}\left(\frac{(2 k-1)^{2}}{4 \epsilon}\right)^{-\frac{1+\alpha}{2}} \sum_{m=0}^{\infty} \frac{\left(\frac{1+\alpha}{2}\right)_{m}\left(\frac{2+\alpha}{2}\right)_{m}}{m !}\left(\frac{(2 k-1)^{2}}{4 \epsilon}\right)^{-m},
$$

up to exponentially small terms. Summing over $k$ we get

$$
\begin{gathered}
\widetilde{Z}(\alpha ; \epsilon ;-1)=\frac{\Gamma\left(\frac{1+\alpha}{2}\right)}{\pi^{\frac{1}{2}+\alpha} \Gamma\left(-\frac{\alpha}{2}\right)} \sum_{m=0}^{\infty} \frac{1}{m !}\left(\frac{1+\alpha}{2}\right)_{m}\left(\frac{2+\alpha}{2}\right)_{m} \zeta\left(2 m+\alpha+1, \frac{1}{2}\right) \epsilon^{m} \\
\quad+\text { exponentially small terms, }
\end{gathered}
$$

where $\zeta(s, q)$ is the Hurwitz zeta function. 
As anticipated, the expansion for the partition function of the $\mathrm{SO}(3)$ Yang-Mills theory does not have any negative or fractional power of $\epsilon$. The coefficients which generalize the $\mathrm{SO}(3)$ intersection numbers in eqs. (3.14) and (3.15), are

$$
a_{m}=\frac{\Gamma\left(\frac{1+\alpha}{2}\right)\left(\frac{1+\alpha}{2}\right)_{m}\left(\frac{2+\alpha}{2}\right)_{m}}{\pi^{\frac{1}{2}+\alpha} \Gamma\left(-\frac{\alpha}{2}\right) m !} \zeta\left(2 m+\alpha+1, \frac{1}{2}\right), \quad m=0,1, \cdots .
$$

We see that $\widetilde{Z}(\alpha ; 0)$ is finite:

$$
a_{0}=\widetilde{Z}(\alpha ; 0)=\frac{\Gamma\left(\frac{1+\alpha}{2}\right)}{\pi^{\frac{1}{2}+\alpha} \Gamma\left(-\frac{\alpha}{2}\right)} \zeta\left(\alpha+1, \frac{1}{2}\right),
$$

even though $\mathcal{M}\left(\overline{\mathcal{S}}_{g, p}\right)$ is expected to be infinite dimensional for non-integer $\alpha$. We believe that in a proper mathematical set up, $a_{0}$ can be interpreted as an appropriately renormalized "symplectic volume". The fact that $\widetilde{Z}(\alpha ; 0)$ is finite only for moduli spaces which are smooth, is a non-trivial consistency check of our proposal, namely that the numbers $a_{m}$ in eqs. (4.10) and (4.17) do indeed encode topological information of some well defined moduli spaces.

\subsubsection{Comparison at negative integer $\alpha$}

As in case of $\mathrm{SU}(2)$, the results of the previous section can be recovered by setting $\alpha=$ $(2-2 g)$ in eq. (4.16). We use the relation $\zeta\left(s, \frac{1}{2}\right)=\left(2^{s}-1\right) \zeta(s)$ between the Hurwitz and the Riemann zeta functions, and use the identities for the zeta and $\Gamma$-functions to write

$$
\begin{gathered}
\widetilde{Z}(\alpha ; \epsilon)=\sum_{\substack{m=0 \\
\\
\quad+\text { exponentially small terms. }}} \frac{(-1)^{m} \pi^{2 m}\left(2^{2 m+3-2 g}-1\right)}{m !} \zeta(2 g-2 m-2) \epsilon^{m}+\frac{(-1)^{g} \pi^{2 g-2}}{2(g-1) !} \epsilon^{g-1} \\
\end{gathered}
$$

The evaluation of the $(g-1)$-th term needed a little care as it involves a cancellation between a simple zero of the Pochhammer symbol, $(2-g)_{m} \sim(-1)^{g-1} \Gamma(g-1)(g-m-1)$ for $m=g-1$, with the simple pole of the zeta function, $\zeta(2 m-2 g-1) \sim \frac{1}{2(m-g+1)}$.

\section{$5 \quad \mathrm{SU}(2)$ theory with a holonomy along a boundary}

In the case of the SU(2) Yang-Mills on a Richards surface without boundary we found that the small $\epsilon$ expansion of the partition function contained a term with fractional power. We think of this as an indication that the moduli space of $\mathrm{SU}(2)$ flat connections on Richards surfaces is singular, as in the case of finite genus surfaces. As expected, no such singularity is found in the case of $\mathrm{SO}(3)$. More generally, we expect that the moduli space of flat $\mathrm{SU}(2)$ connections on a Richards surface with a fixed holonomy (other than identity) along its base boundary must be nonsingular, again as is the case for finite genus surfaces. We will confirm this expectation by carrying out an expansion of the partition function (of the $\mathrm{SU}(2)$ theory for definiteness) in small $\epsilon$ for Richards surface with a fixed nontrivial 
holonomy along its boundary. Moreover, the additional contribution from the holonomy gives a refined generating function, which depends on an additional parameter.

The partition function of $\mathrm{SU}(2)$ Yang-Mills theory on a surface with a boundary can be thought of as a map from the holonomy $U_{0}$ at the boundary to the complex numbers. In particular, for the one parameter subgroup $U_{0}(\theta)=\exp \left(i \theta J_{3}\right)$ (where $0 \leq \theta<2 \pi$ ) we have

$$
Z(\alpha ; \epsilon ; \theta)=\sum_{n=1}^{\infty} n^{\alpha-1} \exp \left(-\epsilon \pi^{2} n^{2}\right) \frac{\sin (n \theta / 2)}{\sin (\theta / 2)},
$$

where $\chi_{n}\left(U_{0}(\theta)\right)=\frac{\sin (n \theta / 2)}{\sin (\theta / 2)}$ is the trace of $U_{0}(\theta)$ in the $n$-dimensional representation. Following in the steps of the previous sections, we write this as

$$
Z(\alpha ; \epsilon ; \theta)=\frac{1}{\left(1+e^{i \pi \alpha}\right) \sin \frac{\theta}{2}} \sum_{n=-\infty}^{\infty} n^{\alpha-1} \exp \left(-\epsilon \pi^{2} n^{2}\right) \sin \left(\frac{n \theta}{2}\right)
$$

and performing a Poisson resummation to get

$$
\begin{aligned}
Z(\alpha ; \epsilon ; \theta)=-\frac{2^{\alpha-2} \Gamma\left(\frac{1+\alpha}{2}\right)}{\epsilon^{\frac{1+\alpha}{2}} \sin (\theta / 2)} \sum_{k=-\infty}^{\infty}\left((4 k \pi-\theta){ }_{1} F_{1}\left(\frac{1+\alpha}{2}, \frac{3}{2},-\frac{(4 \pi k-\theta)^{2}}{4 \epsilon}\right)\right. \\
\left.-(4 k \pi+\theta)_{1} F_{1}\left(\frac{1+\alpha}{2}, \frac{3}{2},-\frac{(4 \pi k+\theta)^{2}}{4 \epsilon}\right)\right) .
\end{aligned}
$$

Using the asymptotic expansion eq. (4.7), $Z(\alpha ; \epsilon ; \theta)$ is

$$
\begin{aligned}
-\frac{2^{\alpha-3} \sqrt{\pi} \Gamma\left(\frac{1+\alpha}{2}\right)}{\epsilon^{\frac{1+\alpha}{2}} \sin \left(\frac{\theta}{2}\right)} \sum_{k=-\infty}^{\infty} & {\left[\frac{(4 \pi k-\theta)(4 \epsilon)^{(1+\alpha) / 2}}{|4 \pi k-\theta|)^{\alpha+1} \Gamma\left(\frac{2-\alpha}{2}\right)} \sum_{m=0}^{\infty} \frac{\left(\frac{\alpha}{2}\right)_{m}\left(\frac{1+\alpha}{2}\right)_{m}}{m !}\left(\frac{4 \epsilon}{(4 \pi k-\theta)^{2}}\right)^{m}\right.} \\
- & \left.\frac{(4 \pi k+\theta)(4 \epsilon)^{(1+\alpha) / 2}}{|4 \pi k+\theta|^{\alpha+1} \Gamma\left(\frac{2-\alpha}{2}\right)} \sum_{m=0}^{\infty} \frac{\left(\frac{\alpha}{2}\right)_{m}\left(\frac{1+\alpha}{2}\right)_{m}}{m !}\left(\frac{4 \epsilon}{(4 \pi k+\theta)^{2}}\right)^{m}\right] \\
& + \text { exponentially small terms }
\end{aligned}
$$

which, after doing the sum over $k$, gives

$$
\begin{aligned}
Z(\alpha ; \epsilon ; \theta)=\frac{2^{1+\alpha} \sqrt{\pi} \Gamma\left(\frac{1+\alpha}{2}\right)}{\sin \left(\frac{\theta}{2}\right) \Gamma\left(-\frac{\alpha}{2}\right)} \sum_{m=0}^{\infty} \frac{\left(\frac{2+\alpha}{2}\right)_{m-1}\left(\frac{1+\alpha}{2}\right)_{m}}{(2 \pi)^{2 m+\alpha} m !} \\
\times\left[\zeta\left(2 m+\alpha, 1-\frac{\theta}{4 \pi}\right)-\zeta\left(2 m+\alpha, \frac{\theta}{4 \pi}\right)\right] \epsilon^{m} .
\end{aligned}
$$

As expected, this expression does not have any fractional power of $\epsilon$.

\section{Conclusions}

The moduli spaces of flat connections on two dimensional surfaces are of considerable importance. Consequently, these have been studied extensively by mathematicians as well as physicists using diverse tools. In particular, the partition function of $2 \mathrm{dYM}$ on surfaces contains information about various intersection numbers of the moduli spaces of flat connections on principal bundles on these surfaces. 
In this paper we made a step towards extending these structures to surfaces of infinite genus. More precisely, we analyzed the weak coupling expansion of the partition function of $2 \mathrm{dYM}$ on a class of such surfaces, which we call Richards surfaces, that have a recursive structure. The partition function of $2 \mathrm{dYM}$ on Richards surfaces was computed in [27]. Here we analyzed in detail its weak coupling expansion for the gauge groups $\mathrm{SU}(2)$ and $\mathrm{SO}(3)$, with and without the insertion of a Wilson line.

We were able to do this by considering the partition function as an analytic function of the complexified Euler characteristic of a surface. Considering the Euler characteristic at fractional values as appropriate to Richards surfaces (or continuing analytically to negative integral values as appropriate for surfaces of finite characteristic), we found that the corresponding partition function can be split into three contributions. First, there is a term which is finite whenever the corresponding theory admits a smooth moduli space of classical solutions at zero coupling. It seems reasonable to interpret this as the symplectic volume of the (infinite dimensional) moduli space of flat connections on Richards surfaces. The fact that it turns out to be finite whenever the moduli space is smooth is a consistency check that this geometrical interpretation is coherent.

Secondly, there is a contribution to the partition function that is perturbative (in $\epsilon$ ). In the finite genus case the perturbative series stops at a finite order. It was understood by Witten [4] that the finite number of perturbative coefficients are the intersection numbers of certain cohomology classes on the (finite-dimensional) moduli space of flat connections on surfaces of finite genus. In the Richards case we discovered that the perturbative expansion never stops. This appears to be consistent with the expected infinite dimensionality of the corresponding moduli space of flat connections. The infinite number of coefficients of this expansion, for which we gave explicit formulas, are therefore the natural candidates for the intersection numbers on some appropriately defined moduli space of flat connections over Richards surfaces. We hope to report on this geometric interpretation in the near future.

Finally, for both the finite and the infinite genus cases, there are non-perturbative, instanton-like corrections which are exponentially small. Although these can be extracted from the expressions we have derived, we have not analyzed these terms in details to connect them to non-flat classical solutions. This exercise, which we leave for the future, may be a rewarding one since it could provide a proper field theoretic context in which to extend the tools and concepts of resurgent analysis which has so far been applied largely to quantum mechanical systems.

\section{Acknowledgments}

It is a pleasure to thank François Labourie for useful discussions. The work of DK is supported by a research fellowship from the Council of Scientific Research (CSIR), India, and that of CI, in part, by the INFN, Italy. DG gratefully acknowledges the hospitality at the Università degli studi di Genova, where this project was initiated during a visit that was supported by the SERP-Chem Master and the Erasmus Mundus programme of the European Union. 
Open Access. This article is distributed under the terms of the Creative Commons Attribution License (CC-BY 4.0), which permits any use, distribution and reproduction in any medium, provided the original author(s) and source are credited.

\section{References}

[1] G. 't Hooft, A two-dimensional model for mesons, Nucl. Phys. B 75 (1974) 461 [INSPIRE].

[2] A.A. Migdal, Recursion equations in gauge theories, Sov. Phys. JETP 42 (1975) 413 [Zh. Eksp. Teor. Fiz. 69 (1975) 810] [INSPIRE].

[3] E. Witten, On quantum gauge theories in two-dimensions, Commun. Math. Phys. 141 (1991) 153 [INSPIRE].

[4] E. Witten, Two-dimensional gauge theories revisited, J. Geom. Phys. 9 (1992) 303 [hep-th/9204083] [INSPIRE].

[5] S. Cordes, G.W. Moore and S. Ramgoolam, Large-N $2 D$ Yang-Mills theory and topological string theory, Commun. Math. Phys. 185 (1997) 543 [hep-th/9402107] [INSPIRE].

[6] C. Vafa, Two dimensional Yang-Mills, black holes and topological strings, hep-th/0406058 [INSPIRE].

[7] S. de Haro and M. Tierz, Brownian motion, Chern-Simons theory and 2D Yang-Mills, Phys. Lett. B 601 (2004) 201 [hep-th/0406093] [INSPIRE].

[8] H. Ooguri, A. Strominger and C. Vafa, Black hole attractors and the topological string, Phys. Rev. D 70 (2004) 106007 [hep-th/0405146] [INSPIRE].

[9] M. Aganagic, H. Ooguri, N. Saulina and C. Vafa, Black holes, q-deformed $2 D$ Yang-Mills and non-perturbative topological strings, Nucl. Phys. B 715 (2005) 304 [hep-th/0411280] [INSPIRE].

[10] N. Caporaso et al., Topological strings, two-dimensional Yang-Mills theory and Chern-Simons theory on torus bundles, Adv. Theor. Math. Phys. 12 (2008) 981 [hep-th/0609129] [INSPIRE].

[11] S. Cordes, G.W. Moore and S. Ramgoolam, Lectures on 2D Yang-Mills theory, equivariant cohomology and topological field theories, Nucl. Phys. Proc. Suppl. 41 (1995) 184 [hep-th/9411210] [INSPIRE].

[12] E. Witten, Supersymmetric Yang-Mills theory on a four manifold, J. Math. Phys. 35 (1994) 5101 [hep-th/9403195] [INSPIRE].

[13] M. Blau and G. Thompson, Localization and diagonalization: a review of functional integral techniques for low dimensional gauge theories and topological field theories, J. Math. Phys. 36 (1995) 2192 [hep-th/9501075] [INSPIRE].

[14] C. Lozano and M. Mariño, Donaldson invariants of product ruled surfaces and two-dimensional gauge theories, Commun. Math. Phys. 220 (2001) 231 [hep-th/9907165] [INSPIRE].

[15] N.A. Nekrasov, Seiberg-Witten prepotential from instanton counting, Adv. Theor. Math. Phys. 7 (2004) 831 [hep-th/0206161] [INSPIRE].

[16] C. Beasley and E. Witten, Non-Abelian localization for Chern-Simons theory, J. Diff. Geom. 70 (2005) 183 [hep-th/0503126] [INSPIRE]. 
[17] S. Giombi, V. Pestun and R. Ricci, Notes on supersymmetric Wilson loops on a two-sphere, JHEP 07 (2010) 088 [arXiv:0905.0665] [INSPIRE].

[18] V. Pestun, Localization of the four-dimensional $N=4 S Y M$ to a two-sphere and $1 / 8$ BPS Wilson loops, JHEP 12 (2012) 067 [arXiv:0906.0638] [INSPIRE].

[19] S. Giombi and V. Pestun, Correlators of local operators and $1 / 8$ BPS Wilson loops on $S^{2}$ from $2 d$ YM and matrix models, JHEP 10 (2010) 033 [arXiv:0906.1572] [INSPIRE].

[20] M.S. Narasimhan and C.S. Seshadri, Stable and unitary vector bundles on a compact Riemann surface, Ann. Math. 82 (1965) 540.

[21] M.F. Atiyah and R. Bott, The Yang-Mills equations over Riemann surfaces, Phil. Trans. Roy. Soc. Lond. A 308 (1982) 523 [INSPIRE].

[22] M. Thaddeus, Conformal field theory and the cohomology of the moduli space of stable bundles, J. Diff. Geom. 35 (1992) 131 [INSPIRE].

[23] C. King and A. Sengupta, The semiclassical limit of the two-dimensional quantum Yang-Mills model, J. Math. Phys. 35 (1994) 5354 [hep-th/9402135] [InSPIRE].

[24] V.V. Fock and A.A. Rosly, Poisson structure on moduli of flat connections on Riemann surfaces and r matrix, Amer. Math. Soc. Transl. 191 (1999) 67 [math/9802054] [INSPIRE].

[25] K.-F. Liu, Mathematical results inspired by physics, math/0304460 [INSPIRE].

[26] A.N. Sengupta, Gauge theory in two dimensions: topological, geometric and probabilistic aspects, arXiv:0707.4136 [INSPIRE].

[27] D. Kumar, Two-dimensional Yang-Mills theory on recursive infinite genus surfaces, JHEP 09 (2014) 023 [arXiv: 1407.6379] [INSPIRE].

[28] I. Richards, On the classification of noncompact surfaces, Trans. Amer. Math. Soc. 106 (1963) 259.

[29] D. Friedan and S.H. Shenker, The analytic geometry of two-dimensional conformal field theory, Nucl. Phys. B 281 (1987) 509 [InSPIRE].

[30] H.S. La, Thermodynamics of handles, Annals Phys. 205 (1991) 458 [INSPIRE]. 